\title{
Associations of Thyroid Hormone Levels and Macrovascular Complications in Euthyroid Type 2 Diabetic Patients
}

\section{Yonghui $\mathrm{Hu}$ \\ Zhiyue Yan \\ Congqing Pan}

NHC Key Laboratory of Hormones and Development, Tianjin Key Laboratory of Metabolic Diseases, Chu Hsien-I Memorial Hospital \& Tianjin Institute of Endocrinology, Tianjin Medical University, Tianjin, People's Republic of China
Correspondence: Congqing Pan NHC Key Laboratory of Hormones and Development, Tianjin Key Laboratory of Metabolic Diseases, Chu Hsien-I Memorial Hospital \& Tianjin Institute of Endocrinology, Tianjin Medical University, Tianjin, People's Republic of China

Tel +86- I3920423976

Fax +86-22-59560475

Email profpancq@163.com
Purpose: The purpose of this study is to evaluate whether thyroid hormone in euthyroid patients with type 2 diabetes mellitus (T2DM) is associated with macrovascular complications.

Patients and Methods: The authors examined 311 patients enrolled from February 2019 to December 2019 in Tianjin Medical University Chu Hsien-I Memorial Hospital. A medical record review enabled the collection of demographic and anthropometric information. We classified the patients into two groups based on the echocardiography and vascular ultrasonography results, namely, non-macrovascular complications $(\mathrm{n}=131)$ group and macrovascular complications $(n=180)$ group. Odds ratios (OR) and $95 \%$ confidence intervals (CI) were calculated, adjusting for potential confounders, the prevalence of macrovascular complications was determined using multivariate logistic regression.

Results: A significant association was observed for diabetic macrovascular complications with normal free triiodothyronine (FT3) $(\mathrm{OR}=0.534,95 \%$ CI $0.358-0.796, \mathrm{p}=0.002)$ and free thyroxine $(\mathrm{FT} 4)(\mathrm{OR}=0.844,95 \% \mathrm{CI} 0.760-0.937, \mathrm{p}=0.001)$. Nevertheless, there was no evidence of any association between thyroid-stimulating hormone (TSH) and the development of diabetic macrovascular complications. When stratified by the body mass index (BMI), a similar relationship existed with the overall results. The positive association remained in restricted analyses involving only patients with HbA1c abnormalities.

Conclusion: Overweight or obese T2DM patients are at high risk due to the implicit association between low but clinically normal thyroid hormone levels and elevated risk of macrovascular complications. However, there were no statistically significant associations between TSH and diabetic macrovascular complications.

Keywords: macrovascular complications, T2DM, thyroid hormone, type 2 diabetes mellitus

\section{Introduction}

Diabetes mellitus (DM) is classified under the group of chronic metabolic diseases. Generally, DM is characterized by hyperglycemia. Besides the impact on the patients' quality of life, DM causes a significant financial burden on patients and the global healthcare system. According to the International Federation of Diabetes, the global number of diabetic people has increased to 425 million, of which $5-10 \%$ are T1DM patients and 90-95\% are T2DM patients. ${ }^{1}$ In 2017 , the prevalence of DM in China was $10.9 \%$ among adults. ${ }^{2}$ Inappropriate therapy or poor alimentary control of DM patients often leads to life-threatening chronic complications, such as diabetic nephropathy, neuropathy, retinopathy, and cardiomyopathy. In China, 
nearly $46 \%$ of outpatients with T2DM have a history of microvascular complications, $10 \%$ have cerebrovascular diseases, and $15 \%$ have cardiovascular disease. ${ }^{3}$ Generally, these chronic complications are divided into two categories: microvascular complications mainly in the kidneys, the eyes, and the nervous system and macrovascular complications primarily involving cardiovascular conditions, such as coronary, cerebrovascular, and peripheral arterial diseases.

The pathophysiology of diabetic complications has not been completely elucidated. However, several clinical studies have revealed some risks, such as duration of diabetes, ${ }^{4}$ hypertension, ${ }^{5}$ the range of HbA1c, glycaemic control, dyslipidaemia, lifestyle, ${ }^{6}$ smoking, vitamin $\mathrm{D}$ deficiency, cystatin $\mathrm{C}^{7}$ etc.

The clinical significance of thyroid hormones is widely recognized for the role in organism physiology and functional development. Therefore, scholars began studying the association between thyroid hormones and cardiovascular disease. In a recent study by Asvold et $\mathrm{al}^{8}$ it was demonstrated that thyrotropin levels within the reference range in women were positively and linearly related to the mortality due to coronary heart disease (CHD). Jung et $\mathrm{al}^{9}$ proposed that higher serum free thyroxine levels have a positive correlation with coronary artery disease. Recently, scholars have started paying attention to the impact of thyroid hormones (THs) on diabetic complications. Several clinical studies have demonstrated that thyroid dysfunction is related to diabetic complications. Subsequentially, more recent studies have extended the association into the euthyroid states. However, these studies have primarily focused on the association between thyroid hormones and diabetic microvascular complications. ${ }^{7,10,11}$ Only a few researchers have studied whether thyroid hormone levels elevate the risk of diabetic macrovascular complications.

The present retrospective study explores the association between macrovascular complications and thyroid functions in euthyroid T2DM patients. The conclusions will enrich the existing literature and generate fresh insights for future studies.

\section{Patients and Methods Study Population}

We retrospectively recruited 311 T2DM patients (180 women and 131 men) at Tianjin Medical University Chu Hsien-I Memorial Hospital from February 2019 to
December 2019. The patients were diagnosed based on the criteria of the American Diabetes Association standard. ${ }^{12}$ The following exclusion criteria were considered: acute complications of diabetes, history of hypothalamus or pituitary diseases, current malignancy, pregnancy, nondiabetic cardiovascular or cerebrovascular problems, urinary tract infection, under any medication that affects thyroid function, missing essential relevant information, autoimmune thyroid diseases and a history of macrovascular related diseases before the diagnosis of diabetes.

\section{Data Collection}

The collected demographic and anthropometric parameters include age, sex, diabetes duration, cardiovascular or cerebrovascular disease history, and history of other diseases. Following overnight fasting, venous blood was drawn from each patient to examine the following laboratory parameters: total cholesterol (TC), triglyceride (TG), highdensity lipoprotein cholesterol (HDL-C), low-density lipoprotein cholesterol (LDL-C), very low-density lipoprotein cholesterol (VLDL-C), glycosylated hemoglobin (HbA1c), free triiodothyronine (FT3), free thyroxine (FT4), and thyroid-stimulating hormone (TSH) levels. The height, body weight, and blood pressure (BP) of all participants were measured. The same physician measured the BP in a seated position after a rest period of more than $5 \mathrm{~min}$.

Normal thyroid function was defined as thyroid hormones in the normal range which was included in this study. The nominal reference range of thyroid hormones is TSH $(0.56-5.91 \mu \mathrm{IU} / \mathrm{mL})$, FT3 $(3.28-6.47 \mathrm{pmol} / \mathrm{L})$, and FT4 (7.64-16.03 pmol/L).

Macrovascular complications were identified as atherosclerosis of the aorta, coronary, basilar, lower limb arteries or carotid arteries based on the diagnoses of echocardiography and vascular ultrasonography.

\section{Statistical Analysis}

In the present study, IBM SPSS26.0 software (SPSS, Inc., Chicago, IL, USA) for Windows was used for all statistical analysis. Each continuous variable was examined for normal distribution. Continuous variables with a normal distribution were presented as the mean (standard deviation, SD). Meanwhile, nonnormal-distributed variables were summarized as the median (interquartile range, IQR). Student's $t$-test or Mann-Whitney $U$-test was performed to compare differences in the variables between two groups as appropriate. Moreover, categorical variables are reported as frequency, and intergroup comparisons 
were conducted using the Chi-square test. Odds ratios (OR) and 95\% confidence intervals (CI) were calculated with multivariate logistic regression to assess the independent associations of thyroid hormones and diabetic macrovascular complications while adjusting for potential confounders (model 1: adjusted for age and sex; model 2: adjusted for all confounders). Since BMI could influence the association between thyroid hormones and diabetes complications, we calculated the stratum-specific ORs for normal or underweight patients $(\mathrm{BMI}<25)$ and overweight or obese patients $(\mathrm{BMI} \geq 25)$ to test the effect modification by BMI. Based on the correlations between HbA1c and the development of atherosclerosis in T2DM patients demonstrated in previous researches, ${ }^{13}$ patients with normal HbA1c $(<6.0 \%)$ were rarely encountered in our study (only 26 patients). Hence, we performed sensitivity analyses by excluding patients with normal HbAlc.

\section{Results}

Our study included a total of 365 patients enrolled from February 2019 to December 2019. Of these cases, 35 cases were excluded owing to preexisting macrovascular complications. Furthermore, 19 cases were missing macrovascular examination reports. Therefore, 311 patients were included in the final analysis. According to the results from macrovascular examinations, 131 patients (42.1\%) were categorized as normal and 180 patients (57.9\%) were categorized with macrovascular complications. Table 1 presents the baseline characteristics of the patients.

Among the 131 females and 180 males, the age (median (IQR)) was 56 (12) years and the BMI was 26.51 (3.98) kg/ $\mathrm{m} 2$. Moreover, 217 patients were subcutaneously injected with insulin to control blood glucose levels. Meanwhile, 94 patients were treated with oral medications as part of antidiabetic therapy. The thyroid hormones including FT3 and FT4 were significantly lower in participants with macrovascular complications compared with those without macrovascular complications. However, there was no significant difference between the two groups regarding the TSH levels.

Table 2 shows the results from the multivariate logistic regression was performed between the prevalence of macrovascular complications and the level of thyroid hormones. A graded decrease in the risk was found in the prevalence of macrovascular complications with the increase of FT3 and FT4. Model 1 (Figure 1A) was adjusted for age and sex. Accordingly, high normal level of FT3 and FT4 revealed lower prevalence ratios of 0.589 (95\% CI $0.407-0.854, \mathrm{p}=$ 0.005 ) and 0.854 (95\% CI 0.773-0.944, p $=0.002)$, respectively. In addition to age and sex, in the model adjusted for duration of T2DM, systolic blood pressure (SBP), diastolic blood pressure (DBP), BMI, HbAlc, TG, TC, HDL-C, LDL-C, and VLDL-C the prevalence ratio of high FT3 levels was $0.534(95 \%$ CI 0.358-0.796, $\mathrm{p}=0.002)$ and high-normal FT4 levels (OR 0.844 (95\% CI 0.760-0.937), $\mathrm{p}=0.001$ ) was smaller than low-normal FT4 levels (Figure 1B). In the comparison of TSH levels, there was no significant difference in the prevalence of macrovascular complications. When stratified by BMI, there was no association in normal weight patients but a similar association was present in the overweight or obese patients (Table 2).

Moreover, sensitivity analyses were conducted to exclude patients with normal HbAlc. The results were generally similar once patients who exhibited normal HbA1c $(n=26)$ were excluded (Table 3$)$. In this group, the association of FT3 was slightly enhanced. However, the association of FT4 was slightly weakened (Figure 2).

\section{Discussion}

The present retrospective study explores the relationship between thyroid hormones and the prevalence of diabetic macrovascular complications. Overall, after adjusting for the potential confounders including age, sex, duration of T2DM, BMI, SBP, DBP, HbA1c, TG, TC, HDL-C, LDL-C, and VLDL-C, the results indicated that low-normal FT3 and FT4 levels are related to an elevated risk of macrovascular complications. As determined by BMI, statistically significant associations were found between free thyroid hormone and diabetic macrovascular complications among overweight or obese patients. There was no association between the thyroid hormone and diabetic macrovascular complications among patients who were normal weight. A similar association was observed among those that exhibited HbA1c levels above the nominal range.

Cardiovascular and cerebrovascular complications are frequently conjoined with T2DM since such complications are the principal causes of morbidity and mortality in T2DM complications. Upon adjusting for known cardiovascular risk factors, the risk of macrovascular complications was still found to be still four times higher in T2DM patients compared to participants without T2DM. ${ }^{14}$ Some endocrine hormones, such as the thyroid hormone, regulated carbohydrate metabolism, which includes the absorption of glucose, hepatic gluconeogenesis, and glycogenolysis. ${ }^{15,16}$ Therefore, thyroid hormones could contribute to the pathogenesis of macrovascular complications in T2DM. 
Table I Demographical and Clinical Characteristics of the Study Population with and without Macrovascular Complications

\begin{tabular}{|c|c|c|c|c|}
\hline \multicolumn{5}{|c|}{ Patients Not Stratified by BMI } \\
\hline Characteristics & $\begin{array}{l}\text { Total } \\
(n=3 I I)\end{array}$ & $\begin{array}{l}\text { Non-Macrovascular Complications } \\
(n=|3|)\end{array}$ & $\begin{array}{l}\text { Macrovascular Complications } \\
(n=180)\end{array}$ & $P$ value \\
\hline Age (years) & $56(12)$ & $54(15)$ & $58(13)$ & $<0.01$ \\
\hline Sex (male/female) & $|80 / 13|$ & $81 / 50$ & $99 / 81$ & 0.228 \\
\hline $\begin{array}{l}\text { Duration of T2DM } \\
\text { (years) }\end{array}$ & $8.00(12.00)$ & $6.00(10.00)$ & $10.00(12.00)$ & 0.001 \\
\hline $\mathrm{SBP}(\mathrm{mmHg})$ & $130(30)$ & $130(20)$ & $138(30)$ & 0.043 \\
\hline $\mathrm{DBP}(\mathrm{mmHg})$ & $80(12)$ & $80(12)$ & $80(10)$ & 0.347 \\
\hline BMI $\left(\mathrm{Kg} / \mathrm{mm}^{2}\right)$ & $26.51 \pm 3.98$ & $26.06 \pm 3.99$ & $26.85 \pm 3.96$ & 0.085 \\
\hline TSH (mIU/L) & $1.83(0.66)$ & $1.89(0.88)$ & $1.79(0.68)$ & 0.254 \\
\hline FT3 (pmol/L) & $4.42(0.94)$ & $4.58(0.83)$ & $4.30(0.99)$ & 0.002 \\
\hline FT4 (pmol/L) & $12.53(4.20)$ & $13.47(3.12)$ & $12.27(5.49)$ & 0.001 \\
\hline HbAlc (\%) & $8.90(3.10)$ & $9.16(3.00)$ & $8.70(2.70)$ & 0.009 \\
\hline TG (mmol/L) & $1.89(1.50)$ & $2.25(1.46)$ & $1.70(1.53)$ & 0.055 \\
\hline $\mathrm{TC}(\mathrm{mmol} / \mathrm{L})$ & $5.08(1.35)$ & $5.08(1.02)$ & $5.08(1.56)$ & 0.435 \\
\hline HDL-C (mmol/L) & $1.17(0.31)$ & $1.13(0.33)$ & $1.17(0.30)$ & 0.025 \\
\hline LDL-C (mmol/L) & $3.26(1.00)$ & $3.26(0.83)$ & $3.24(1.19)$ & 0.244 \\
\hline VLDL-C (mmol/L) & $0.63(0.34)$ & $0.66(0.32)$ & $0.60(0.34)$ & 0.072 \\
\hline Insulin therapy (yes/no) & $217 / 94$ & $91 / 40$ & $126 / 54$ & 0.919 \\
\hline \multicolumn{5}{|c|}{ Patients with $\mathrm{BMI}<25 \mathrm{Kg} / \mathrm{mm}^{2}$} \\
\hline Characteristics & Total $(n=8 I)$ & $\begin{array}{l}\text { Non-Macrovascular Complications } \\
(n=42)\end{array}$ & $\begin{array}{l}\text { Macrovascular Complications } \\
(n=39)\end{array}$ & $P$ value \\
\hline Age (years) & $55.95 \pm 10.32$ & $51.48 \pm 10.85$ & $60.77 \pm 7.16$ & $<0.01$ \\
\hline Sex (male/female) & $45 / 36$ & $25 / 17$ & $20 / 19$ & 0.456 \\
\hline $\begin{array}{l}\text { Duration of T2DM } \\
\text { (years) }\end{array}$ & $6.00(11.00)$ & $5.00(9.50)$ & $9.00(13.00)$ & 0.131 \\
\hline $\mathrm{SBP}(\mathrm{mmHg})$ & $130(24)$ & $122.50(21)$ & $130(30)$ & 0.069 \\
\hline $\mathrm{DBP}(\mathrm{mmHg})$ & $80(1 \mathrm{I})$ & $80(13)$ & $80(10)$ & 0.528 \\
\hline $\mathrm{BMI}\left(\mathrm{Kg} / \mathrm{mm}^{2}\right)$ & $21.68 \pm 1.77$ & $21.68 \pm 1.81$ & $21.68 \pm 1.76$ & 0.997 \\
\hline TSH (mIU/L) & $1.93(0.77)$ & I.9I (0.88) & $1.93(0.76)$ & 0.510 \\
\hline FT3 (pmol/L) & $4.42 \pm 0.70$ & $4.56 \pm 0.64$ & $4.27 \pm 0.75$ & 0.059 \\
\hline FT4 (pmol/L) & $12.75(3.85)$ & I3.85 (3.27) & $12.27(5.20)$ & 0.030 \\
\hline HbAlc (\%) & $9.16(4.00)$ & $9.16(4.02)$ & $9.40(4.00)$ & 0.493 \\
\hline TG (mmol/L) & I.5I (I.5I) & $\mathrm{I} .54$ (I.49) & $1.50(1.34)$ & 0.653 \\
\hline TC (mmol/L) & $5.07(1.18)$ & $5.08(0.7 I)$ & 4.97 (I.78) & 0.613 \\
\hline
\end{tabular}

(Continued) 
Table I (Continued).

\begin{tabular}{|c|c|c|c|c|}
\hline HDL-C (mmol/L) & $1.20(0.40)$ & $1.20 \pm 0.25$ & $1.27 \pm 0.30$ & 0.258 \\
\hline LDL-C (mmol/L) & $3.26(1.13)$ & $3.26(0.72)$ & $3.25(1.29)$ & 0.577 \\
\hline VLDL-C (mmol/L) & $0.55(0.29)$ & $0.56(0.27)$ & $0.50(0.3 \mathrm{I})$ & 0.519 \\
\hline Insulin therapy (yes/no) & $56 / 25$ & $27 / 15$ & $29 / 10$ & 0.327 \\
\hline \multicolumn{5}{|c|}{ Patients with $\mathrm{BMI} \geq 25 \mathrm{Kg} / \mathrm{mm}^{2}$} \\
\hline Characteristics & $\begin{array}{l}\text { Total } \\
(n=230)\end{array}$ & $\begin{array}{l}\text { Non-Macrovascular Complications } \\
(n=89)\end{array}$ & $\begin{array}{l}\text { Macrovascular Complications } \\
(n=14 I)\end{array}$ & $P$ value \\
\hline Age (years) & $56(12)$ & $54(16)$ & $58(12)$ & 0.001 \\
\hline Sex (male/female) & $135 / 95$ & $56 / 33$ & $79 / 62$ & 0.301 \\
\hline $\begin{array}{l}\text { Duration of T2DM } \\
\text { (years) }\end{array}$ & $10.00(12.00)$ & $7.00(10.50)$ & $10.00(12.00)$ & 0.006 \\
\hline SBP $(\mathrm{mmHg})$ & $135.5(26)$ & $130(20)$ & $140(28)$ & 0.356 \\
\hline $\mathrm{DBP}(\mathrm{mmHg})$ & $80(10)$ & $80(10)$ & $80(10)$ & 0.725 \\
\hline BMI $\left(\mathrm{Kg} / \mathrm{mm}^{2}\right)$ & $27.58(3.70)$ & $27.56(3.73)$ & $27.60(3.59)$ & 0.704 \\
\hline TSH (mIU/L) & $1.82(0.64)$ & $1.89(0.95)$ & $1.76(0.65)$ & 0.315 \\
\hline FT3 (pmol/L) & $4.45(0.94)$ & $4.58(0.81)$ & $4.30(0.93)$ & 0.011 \\
\hline FT4 (pmol/L) & $12.37(4.25)$ & $|3.3|(3.09)$ & I2.27 (5.59) & 0.006 \\
\hline HbAlc (\%) & $8.70(2.70)$ & $9.16(2.50)$ & $8.50(2.70)$ & 0.001 \\
\hline $\mathrm{TG}(\mathrm{mmol} / \mathrm{L})$ & $2.10(1.66)$ & $2.39(1.60)$ & $1.76(1.66)$ & 0.012 \\
\hline TC (mmol/L) & $5.08(1.43)$ & $5.08(1.11)$ & $5.08(1.61)$ & 0.490 \\
\hline HDL-C (mmol/L) & $1.15(0.30)$ & $1.10(0.32)$ & $1.17(0.30)$ & 0.017 \\
\hline LDL-C (mmol/L) & $3.25(1.00)$ & $3.26(0.89)$ & $3.23(1.14)$ & 0.256 \\
\hline VLDL-C (mmol/L) & $0.66(0.34)$ & $0.66(0.27)$ & $0.61(0.37)$ & 0.043 \\
\hline Insulin therapy (yes/no) & $161 / 69$ & $64 / 25$ & $97 / 44$ & 0.616 \\
\hline
\end{tabular}

Note: Data are expressed as mean (SD), median (Interquartile range, IQR), or count (percentage) depending on the variable type.

Abbreviations: BMI, body mass index; TC, total cholesterol; TG, triglyceride; HDL-C, high-density lipoprotein cholesterol; LDL-C, low-density lipoprotein cholesterol; VLDL-C, very low-density lipoprotein cholesterol; HbAlc, glycosylated hemoglobin; FT3, free triiodothyronine; FT4, free thyroxine (FT4); TSH, thyroid-stimulating hormone; SBP, systolic blood pressure; DBP, diastolic blood pressure; DM, diabetes mellitus.

Reportedly, low thyroid hormone levels, even within the normal reference range, are related to macrovascular complications. In a prospective study, it was revealed that high TSH and HbA1c within the reference range were corisk factors for the incidence of coronary heart disease. ${ }^{17}$ In a cross-sectional study reported by Gomez-Zamudio et $\mathrm{al}^{18}{ }^{1}$ it was shown that high levels of TSH were associated with elevated pro-inflammatory and cardiovascular risk markers in patients with extreme obesity (BMI $\geq 40$ ), which increases the risk for developing cardiovascular diseases. An association was observed between thyrotropin levels and fatal coronary heart disease (CHD) in a prospective study involving 25,313 cases in NordTrøndelag County in Norway. ${ }^{8}$ Another prospective population-based study in Norway measured thyroid hormone levels in 26,707 cases, and it was found that high-normal TSH levels were associated with an elevated risk of coronary death in females, but not in men. However, thyroid function was not associated with the risk of being hospitalized with myocardial infarction. ${ }^{19}$ Still, none of the studies analyzed the association between macrovascular disease and FT3 or FT4. Moreover, a retrospective study 
Table 2 Prevalence Ratio of Macrovascular Complications Based on Multivariate Logistic Regression on Thyroid Hormone Levels

\begin{tabular}{|c|c|c|c|c|}
\hline \multirow[t]{2}{*}{ Thyroid Status } & \multicolumn{2}{|c|}{ Model I } & \multicolumn{2}{|c|}{ Model 2} \\
\hline & OR $(95 \% \mathrm{CI})$ & $P$ value & OR $(95 \% \mathrm{CI})$ & $P$ value \\
\hline \multicolumn{5}{|c|}{ Patients not stratified by BMI } \\
\hline TSH & $0.835(0.632-1.103)$ & 0.205 & $0.816(0.608-1.094)$ & 0.174 \\
\hline FT3 & $0.589(0.407-0.854)$ & 0.005 & $0.534(0.358-0.796)$ & 0.002 \\
\hline FT4 & $0.854(0.773-0.944)$ & 0.002 & $0.844(0.760-0.937)$ & 0.001 \\
\hline \multicolumn{5}{|c|}{ Patients with $\mathrm{BMl}<25 \mathrm{Kg} / \mathrm{m} 2$} \\
\hline TSH & $0.74 \mid(0.386-1.424)$ & 0.369 & $0.677(0.30 \mathrm{I}-\mathrm{I} .524)$ & 0.347 \\
\hline FT3 & $0.485(0.218-1.083)$ & 0.078 & $0.497(0.202-1.221)$ & 0.127 \\
\hline FT4 & $0.850(0.688-1.049)$ & 0.131 & $0.793(0.614-1.024)$ & 0.075 \\
\hline \multicolumn{5}{|c|}{ Patients with $\mathrm{BMI} \geq 25 \mathrm{Kg} / \mathrm{m}^{2}$} \\
\hline TSH & $0.824(0.601-1.124)$ & 0.222 & $0.816(0.589-1.129)$ & 0.219 \\
\hline FT3 & $0.601(0.390-0.926)$ & 0.021 & $0.568(0.358-0.901)$ & 0.016 \\
\hline FT4 & $0.862(0.768-0.967)$ & 0.012 & $0.853(0.754-0.964)$ & 0.011 \\
\hline
\end{tabular}

Notes: Model I: adjusted for age and sex. Model 2: adjusted for age, sex, duration of T2DM, SBP, DBP, BMI, HbAIc, TG, TC, HDL-C, LDL-C, and VLDL-C. Abbreviations: OR, odds ratio; $95 \% \mathrm{Cl}, 95 \%$ confidence interval.

of 204 cases of coronary artery disease diagnosed by coronary angiograms due to chest pain in Kangbuk Samsung hospital found that elevated levels of serum FT4, even within the nominal range could contribute to coronary artery disease but without any association between TSH and the coronary artery disease. ${ }^{9}$ It is clear that even though some previous studies have examined the association between thyroid hormone and macrovascular diseases, the results are inconsistent. Our research restricted the subjects to patients with T2DM and obtained different results.

In addition, some other studies have shown some limited evidence of a significant association between thyroid hormone and macrovascular complications in T2DM.
A

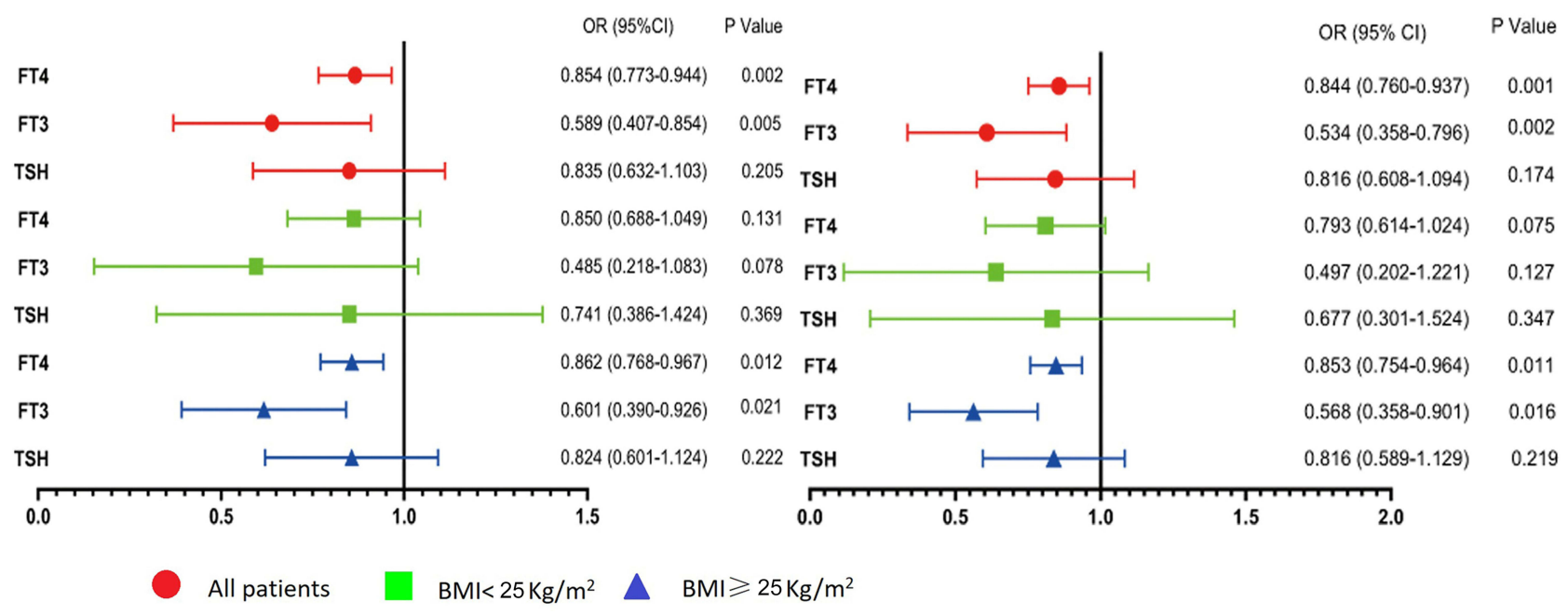

Figure I Prevalence ratio of macrovascular complications based on multivariate logistic regression on thyroid hormone levels. ((A) Adjusted for age and sex. (B) Adjusted for age, sex, duration of T2DM, SBP, DBP, BMI, HbAIc, TG, TC, HDL-C, LDL-C, and VLDL-C.). 
Table 3 Prevalence Ratio of Macrovascular Complications Based on Multivariate Logistic Regression on Thyroid Hormone Levels Within Patients with Abnormal HbAlcHbAlc

\begin{tabular}{|c|c|c|c|c|}
\hline \multirow[t]{2}{*}{ Thyroid Status } & \multicolumn{2}{|c|}{ Model I } & \multicolumn{2}{|c|}{ Model 2} \\
\hline & OR $(95 \% \mathrm{Cl})$ & $P$ value & OR $(95 \% \mathrm{CI})$ & $P$ value \\
\hline \multicolumn{5}{|c|}{ Patients not stratified by BMI } \\
\hline TSH & $0.889(0.666-1.188)$ & 0.428 & $0.880(0.649-1.192)$ & 0.408 \\
\hline FT3 & $0.616(0.420-0.904)$ & 0.013 & $0.546(0.358-0.828)$ & 0.004 \\
\hline FT4 & $0.839(0.755-0.933)$ & 0.001 & $0.823(0.760-0.920)$ & 0.001 \\
\hline \multicolumn{5}{|c|}{ Patients with $\mathrm{BMl}<25 \mathrm{Kg} / \mathrm{mm}^{2}$} \\
\hline TSH & $0.854(0.409-1.784)$ & 0.674 & $0.776(0.313-1.927)$ & 0.585 \\
\hline FT3 & $0.524(0.226-1.215)$ & 0.132 & $0.519(0.199-1.350)$ & 0.178 \\
\hline FT4 & $0.850(0.683-1.057)$ & 0.144 & $0.800(0.619-1.034)$ & 0.088 \\
\hline \multicolumn{5}{|c|}{ Patients with $\mathrm{BMI} \geq 25 \mathrm{Kg} / \mathrm{mm}^{2}$} \\
\hline TSH & $0.857(0.623-1.179)$ & 0.342 & $0.846(0.606-1.181)$ & 0.325 \\
\hline FT3 & $0.627(0.402-0.978)$ & 0.040 & $0.582(0.360-0.940)$ & 0.027 \\
\hline FT4 & $0.845(0.748-0.956)$ & 0.007 & $0.829(0.727-0.945)$ & 0.005 \\
\hline
\end{tabular}

Notes: Model I: adjusted for age and sex. Model 2: adjusted for age, sex, duration of T2DM, SBP, DBP, BMI, HbAIc, TG, TC, HDL-C, LDL-C, and VLDL-C. Abbreviations: OR, odds ratio; $95 \% \mathrm{Cl}, 95 \%$ confidence interval.

Admittedly, methods of evaluating exposure slightly differ from the present study. However, a case-control study conducted in China suggested that low-normal FT3 and FT4 levels contributed to a high prevalence of atherosclerosis after adjusting for covariates. ${ }^{13}$ However, TSH and FT3 to FT4 ratio did not show any significant association with the development of atherosclerosis. Moreover, they analyzed the association of thyroid function with $\mathrm{HbAlc}$ and the association of $\mathrm{HbAlc}$ with the prevalence of atherosclerosis. After adjusting for confounding factors, the result of quantile regression indicated a significant negative association between $\mathrm{HbAlc}$ and
A

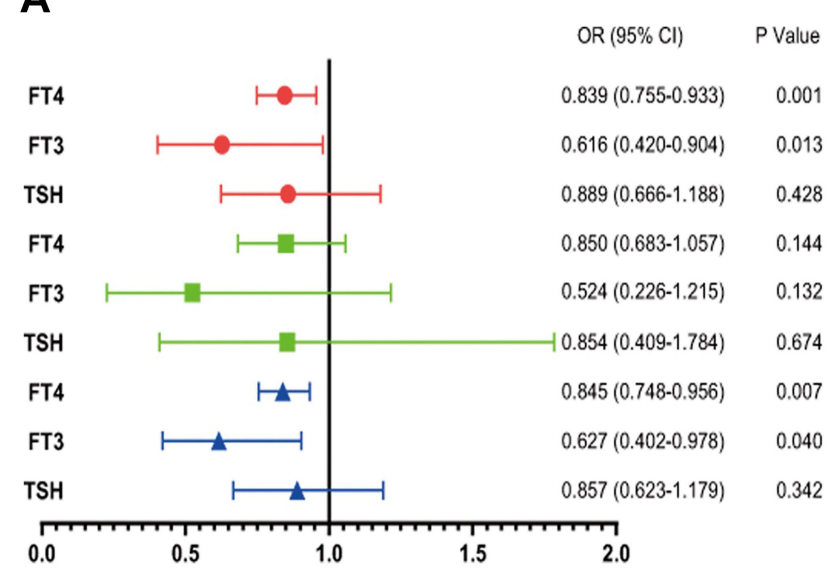

B

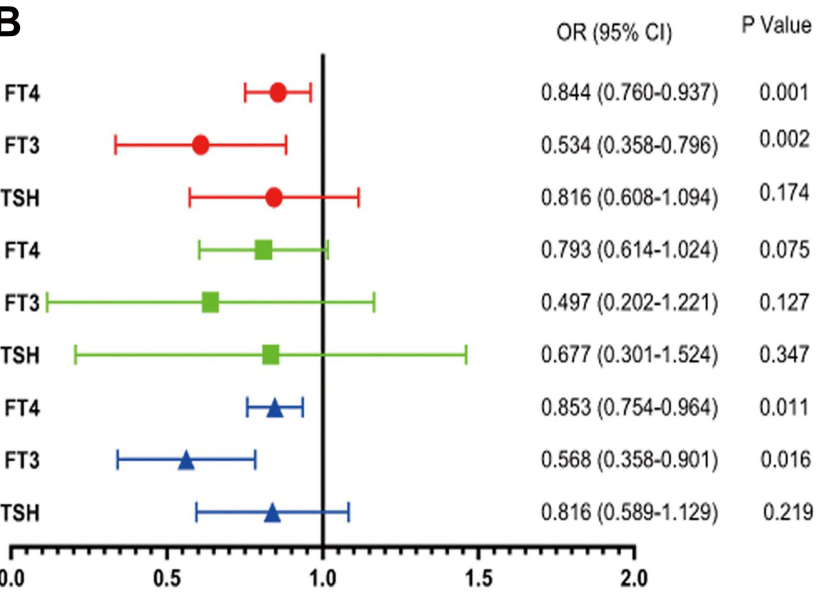

All patients

$\mathrm{BMI}<25 \mathrm{Kg} / \mathrm{m}^{2}$

$\mathrm{BMI} \geqslant 25 \mathrm{Kg} / \mathrm{m}^{2}$

Figure 2 Prevalence ratio of macrovascular complications based on multivariate logistic regression on thyroid hormone levels within patients with abnormal $\mathrm{HbA}$ lc. ((A) Adjusted for age and sex. (B) Adjusted for age, sex, duration of T2DM, SBP, DBP, BMI, HbAlc, TG, TC, HDL-C, LDL-C, and VLDL-C.). 
FT3. However, an opposite trend was observed between HbA1c and FT4. In the analysis of TSH, the confidence intervals of the coefficients between these two included zeros, which did not indicate any significant relationship. In contrast with previous studies, the authors did not find any correlation between $\mathrm{HbAlc}$ and the prevalence of atherosclerosis in the euthyroid T2DM population. The present study is based on the results of previous researches; accordingly, a sensitivity analysis was conducted that involved patients with abnormal HbAlc. In general, similar results were found when observing all included patients. Another study of cross-sectional cohort included 70 cases with T2DM and 70 cases as a control group. It was found that total thyroid hormone instead of free thyroid hormone or TSH was independent of the risk factors associated with cardiovascular events (CVE) in patients with T2DM. ${ }^{20}$ Due to data limitations, the present study did not include total thyroid hormone, which was one of the limitations of the current study. Moreover, thyroid hormone abnormalities contribute to inflammatory activity and CVE in these patients. It is a result that was reflected in previous studies. ${ }^{21-23}$

BMI classified as overweight or obese ( $\mathrm{BMI} \geq 25)$ is widely recognized as a risk factor for developing diabetic macrovascular complications. ${ }^{24}$ Several previous studies have shown that thyroid hormones have a higher level of correlation with cardiovascular disease in obese patients. $^{25-27}$ As we know, this is the first study focused on investigating the association between thyroid hormone and diabetic macrovascular complications based on weight. Elevated risks of diabetic macrovascular complications were observed among high-BMI patients with free thyroid hormone compared to those with normal BMIs, a plausible explanation is that free thyroid hormone contributes to the extra risk of diabetic macrovascular complications among patients already facing a high risk of diabetic macrovascular complications owing to obesity or overweight. It is supported by our finding where the risk increased among overweight or obese patients in the analyses restricted to patients with abnormal $\mathrm{HbAlc}$.

Firstly, the single-center retrospective design was the primary limitation of the present study, which could magnify the confounding. However, the impact was limited by rich covariate data and sensitivity analysis. Secondly, the sample size was inadequate. Therefore, larger, prospective researches are needed to confirm our findings. Moreover, the present research did not show any specific or defined measurements of the macrovascular complications, instead, these were classified according to the echocardiography and vascular cardiography results, which could lead to the overdiagnosis of macrovascular complications $(57.9 \%)$. However, due to the non-invasive nature and highly accurate diagnosis, Doppler ultrasound is currently the preferred method for diagnosing vascular diseases in China. Previous studies have also reported a high incidence of macrovascular complications in T2DM. ${ }^{28-30}$

\section{Conclusions}

The present study found some indicators that free thyroid hormone could decrease the risk of diabetic macrovascular complications. However, there was no strong evidence of any associations between thyroid hormone in T2DM and the risk of macrovascular complications among euthyroid patients with normal weight. Combined with the suggestion, recognizing these types of potential risk factors are significant for reducing the burden from this T2DM complication. Additional longitudinal studies are needed to confirm the findings.

\section{Ethical Approval}

This study was approved by the ethical committee of Tianjin Medical University Chu Hsien-I Memorial Hospital and conducted in accordance with the declaration of Helsinki. The ethical committee waived the requirement of written informed consent for the participants due to the retrospective nature of the study. According to the policy of our hospital, the data was maintained with confidentiality. If anyone wants to access data, should be directed to contact profpancq@163.com.

\section{Acknowledgments}

We thank all colleagues for their help in this study.

\section{Disclosure}

The authors report no conflicts of interest in this work.

\section{References}

1. Gan Q, Wang J, Hu J, et al. The role of diosgenin in diabetes and diabetic complications. J Steroid Biochem Mol Biol. 2020;198:105575. doi:10.1016/j.jsbmb.2019.105575

2. Gu S, Wang X, Shi L, et al. Health-related quality of life of type 2 diabetes patients hospitalized for a diabetes-related complication. Qual Life Res. 2020;29(10):2695-2704. doi:10.1007/s11136-020-02524-3

3. Ji L, Hu D, Pan C, et al. Primacy of the 3B approach to control risk factors for cardiovascular disease in type 2 diabetes patients. $\mathrm{Am}$ $J$ Med. 2013;126(10):925 e911-922. doi:10.1016/j.amjmed.2013. 02.035 
4. Faselis C, Katsimardou A, Imprialos K, Deligkaris P, Kallistratos M, Dimitriadis K. Microvascular complications of type 2 diabetes mellitus. Curr Vasc Pharmacol. 2020;18(2):117-124. doi:10.2174/ 1570161117666190502103733

5. Zoungas S, Woodward M, Li Q, et al. Impact of age, age at diagnosis and duration of diabetes on the risk of macrovascular and microvascular complications and death in type 2 diabetes. Diabetologia. 2014;57(12):2465-2474. doi:10.1007/s00125-014-3369-7

6. Yamazaki D, Hitomi H, Nishiyama A. Hypertension with diabetes mellitus complications. Hypertension Res. 2018;41(3):147-156. doi:10.1038/s41440-017-0008-y

7. Zhao W, Zeng H, Zhang X, et al. A high thyroid stimulating hormone level is associated with diabetic peripheral neuropathy in type 2 diabetes patients. Diabetes Res Clin Pract. 2016;115:122-129. doi:10.1016/j.diabres.2016.01.018

8. Asvold BO, Bjoro T, Nilsen TIL, Gunnell D, Vatten LJ. Thyrotropin levels and risk of fatal coronary heart disease: the HUNT study. Arch Intern Med. 2008;168(8):855-860. doi:10.1001/archinte.168.8.855

9. Jung CH, Rhee EJ, Shin HS, et al. Higher serum free thyroxine levels are associated with coronary artery disease. Endocr J. 2008;55 (5):819-826. doi:10.1507/endocrj.K08E-010

10. Williams GA, Scott IU, Haller JA, Maguire AM, Marcus D, McDonald HR. Single-field fundus photography for diabetic retinopathy screening: a report by the American Academy of Ophthalmology. Ophthalmology. 2004;111(5):1055-1062. doi:10.1016/j.ophtha.2004.02.004

11. Wu J, Li X, Tao Y, Wang Y, Peng Y. Free triiodothyronine levels are associated with diabetic nephropathy in euthyroid patients with type 2 diabetes. Int J Endocrinol. 2015;2015:204893. doi:10.1155/2015/204893

12. American Diabetes Association. Standards of medical care in diabetes--2014. Diabetes Care. 2014;37(Suppl 1):S14-S80.

13. Wang L, Chen T, Yu J, Yuan H, Deng X, Zhao Z. Clinical associations of thyroid hormone levels with the risk of atherosclerosis in euthyroid type 2 diabetic patients in central China. Int J Endocrinol. 2020;2020:2172781. doi:10.1155/2020/2172781

14. Buyken AE, von Eckardstein A, Schulte H, Cullen P, Assmann G. Type 2 diabetes mellitus and risk of coronary heart disease: results of the 10-year follow-up of the PROCAM study. Eur J Cardiovasc Prev Rehabil. 2007;14(2):230-236. doi:10.1097/HJR.0b013e3280142037

15. Korzeniowska KA, Brzezinski M, Szarejko K, et al. The association of thyroid-stimulating hormone (TSH) and free thyroxine (fT4) concentration levels with carbohydrate and lipid metabolism in obese and overweight teenagers. Endokrynol Pol. 2019;70(2):172-178. doi:10.5603/EP.a2018.0090

16. Sinha RA, Singh BK, Yen PM. Thyroid hormone regulation of hepatic lipid and carbohydrate metabolism. Trends Endocrinol Metab. 2014;25(10):538-545. doi:10.1016/j.tem.2014.07.001

17. Li H, Cui Y, Zhu Y, Yan H, Xu W. Association of high normal HbAlc and TSH levels with the risk of CHD: a 10-year cohort study and SVM analysis. Sci Rep. 2017;7:45406. doi:10.1038/srep45406
18. Gomez-Zamudio JH, Mendoza-Zubieta V, Ferreira-Hermosillo A, et al. High thyroid-stimulating hormone levels increase proinflammatory and cardiovascular markers in patients with extreme obesity. Arch Med Res. 2016;47(6):476-482. doi:10.1016/j.arcmed.2016.10.007

19. Asvold BO, Bjoro T, Platou C, Vatten LJ. Thyroid function and the risk of coronary heart disease: 12-year follow-up of the HUNT study in Norway. Clin Endocrinol. 2012;77(6):911-917. doi:10.1111/ j.1365-2265.2012.04477.x

20. Moura Neto A, Parisi MC, Tambascia MA, Pavin EJ, Alegre SM, Zantut-Wittmann DE. Relationship of thyroid hormone levels and cardiovascular events in patients with type 2 diabetes. Endocrine. 2014;45(1):84-91. doi:10.1007/s12020-013-9938-6

21. Boelen A, Kwakkel J, Fliers E. Beyond low plasma T3: local thyroid hormone metabolism during inflammation and infection. Endocr Rev. 2011;32(5):670-693. doi:10.1210/er.2011-0007

22. Wajner SM, Maia AL. New insights toward the acute non-thyroidal illness syndrome. Front Endocrinol. 2012;3:8. doi:10.3389/fendo.2012.00008

23. Yang $\mathrm{T}$, Chu $\mathrm{CH}$, Hsieh $\mathrm{PC}$, et al. C-reactive protein concentration as a significant correlate for metabolic syndrome: a Chinese population-based study. Endocrine. 2013;43(2):351-359. doi:10.1007/s12020-012-9743-7

24. Litwak L, Goh SY, Hussein Z, Malek R, Prusty V, Khamseh ME. Prevalence of diabetes complications in people with type 2 diabetes mellitus and its association with baseline characteristics in the multinational A1chieve study. Diabetol Metab Syndr. 2013;5(1):57. doi:10.1186/1758-5996-5-57

25. Ciccone MM, De Pergola G, Porcelli MT, et al. Increased carotid IMT in overweight and obese women affected by hashimoto's thyroiditis: an adiposity and autoimmune linkage? BMC Cardiovasc Disord. 2010;10:22. doi:10.1186/1471-2261-10-22

26. Brienza C, Grandone A, Di Salvo G, et al. Subclinical hypothyroidism and myocardial function in obese children. Nutr Metab Cardiovasc Dis. 2013;23(9):898-902. doi:10.1016/j.numecd.2012.04.006

27. Gallistl S, Sudi KM, Leschnik B, Muntean W, Borkenstein MH. Inverse correlation between thyroid function and hemostatic markers for coronary heart disease in obese children and adolescents. J Pediatr Endocrinol Metab. 2000;13(9):1615-1620. doi:10.1515/ JPEM.2000.13.9.1615

28. Luijks H, Schermer T, Bor H, et al. Prevalence and incidence density rates of chronic comorbidity in type 2 diabetes patients: an exploratory cohort study. BMC Med. 2012;10:128. doi:10.1186/1741-7015-10-128

29. Song SH, Hardisty CA. Early onset type 2 diabetes mellitus: a harbinger for complications in later years--clinical observation from a secondary care cohort. QJM. 2009;102(11):799-806. doi:10.1093/qjmed/hcp121

30. Zhang X, Decker FH, Luo H, et al. Trends in the prevalence and comorbidities of diabetes mellitus in nursing home residents in the United States: 1995-2004. J Am Geriatr Soc. 2010;58(4):724-730. doi:10.1111/j.1532-5415.2010.02786.x

Diabetes, Metabolic Syndrome and Obesity: Targets and Therapy

Dovepress

\section{Publish your work in this journal}

Diabetes, Metabolic Syndrome and Obesity: Targets and Therapy is an international, peer-reviewed open-access journal committed to the rapid publication of the latest laboratory and clinical findings in the fields of diabetes, metabolic syndrome and obesity research. Original research, review, case reports, hypothesis formation, expert opinion and commentaries are all considered for publication. The manuscript management system is completely online and includes a very quick and fair peer-review system, which is all easy to use. Visit http://www.dovepress.com/testimonials.php to read real quotes from published authors. 